\title{
Discrete computation using a perturbed heteroclinic network
}

\author{
Peter Ashwin and Jon Borresen \\ Department of Mathematical Sciences, University of Exeter, Exeter EX4 4QE, UK
}

\begin{abstract}
Transient synchronization into clusters appears in many biological and physical systems and seems to be important for computation within neural systems. In this paper we show how one can robustly and effectively perform practical computations using small perturbations to a very simple globally coupled network of oscillators. Computations are performed by exploiting the spatio-temporal dynamics of a robust attracting heteroclinic network (also referred to as 'winnerless competition' dynamics) to describe the states and transitions between them. We use different cluster synchronization states to encode states and use this to design a simple multi-base counter. The simulations indicate that this gives a robust computational system exploiting the natural dynamics of the system.
\end{abstract}

Key words: Coupled Oscillator, Neural Computing, Winnerless Competition, Robust Heteroclinic Attractor

PACS: 05.45.Xt, 87.10.+e

\section{Introduction}

Recent experimental and theoretical work by several authors has highlighted the crucial role of spatio-temporal dynamics in neural systems. In particular, work on olfactory systems of insects and fish has demonstrated that recognized odors manifest themselves as different spatio-temporal outputs from certain sub-systems such as $[1,3,2,4]$ for insect antennal lobes (AL) and $[5,6]$ for the Zebrafish olfactory bulb (OB). It has been suggested that the dynamics of the network responsible for the transformation of input to output of the AL/OB for these systems is one of 'winnerless competition' $[1,7-9]$; this is a type of attracting dynamics consisting of saddle periodic orbits linked together by unstable manifolds. Similar switching behaviour has been observed in other highly coupled systems, for example between competing modes in multimode 
laser systems $[10,11]$. Such attractors may seem at first unnatural but in systems that are close to symmetric, and in systems that have invariant subspaces they may be robust; i.e. structurally stable to perturbations that preserve the symmetric or invariant subspace structure. Such attractors have been found in a range of physically relevant models and experiments [12-15].

The AL/OB system is far from being a simple relay; it shows effects such as short term memory, anticipation and computation [7]. The aim of this paper is to show that a globally coupled system idealizing the AL/OB architecture can be used to explicitly design computational systems by exploiting robust heteroclinic attractors (winnerless competition). We use globally coupled phase oscillators that exhibit slow switching between cluster states [16-19]. Previous work [20] has shown that robust heteroclinic attractors for a particular system of five globally coupled phase oscillators allow one to encode up to twenty different states as synchronized clusters, and moreover to move between them along heteroclinic orbits. In this paper we show how one can practically use the dynamics analysed in [20] to perform discrete computations. In doing so, we address issues of response of the system to noise, detection of cluster states and timing as well as giving an example of a network that exploits the dynamics to act as a simple multi-base counter.

\section{Globally coupled oscillator dynamics: heteroclinic networks}

We consider perturbations to the model of Hansel et al. [16] for $N$ globally coupled oscillators with phases $\theta_{i} \in \mathbf{T}=[0,2 \pi)$ given by

$$
\dot{\theta}_{i}=\omega+\frac{1}{N} \sum_{j=1}^{N} g\left(\theta_{i}-\theta_{j}\right)+\eta w_{i}(t)+\epsilon I_{i}(t)
$$

where $i=1, \cdots, N$ and $g(\phi)=-\sin (\phi+\alpha)+r \sin (2 \phi)$; see also [17-19]. The quantities $w_{i}(t)$ represent derivatives of independent, identically distributed Brownian processes with zero mean and unit variance per unit time. The inputs $\left|I_{i}(t)\right| \leq 1$ are used to control the state of the system. We include $\eta$ and $\epsilon$, small parameters that control the strength of the noise and perturbations respectively.

The unperturbed system $(1)(\eta=\epsilon=0)$ has symmetry $\mathbf{S}_{N}$ corresponding to permutations of the $N$ oscillators. Using these symmetries one can identify robust heteroclinic attractors (first noted theoretically in [13] and then practically for this example in [16]) that exist for open sets of parameters. These attractors consist of saddle periodic orbits of symmetry $\mathbf{S}_{k} \times \mathbf{S}_{N-k}$ and their unstable manifolds. On the periodic orbit there are only two possible phases

of an oscillator at any point in time: precisely $k$ of them are at one phase and 


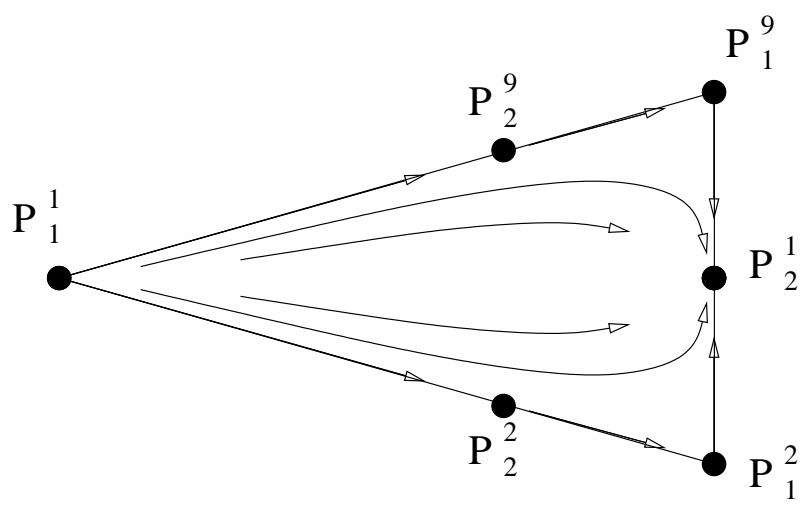

Fig. 1. Schematic showing part of the two dimensional unstable manifold $W^{u}\left(P_{1}^{1}\right)$ almost all trajectories leaving from $P_{1}^{1}$ converge to $P_{2}^{1}$ but exceptional trajectories converge to $P_{2}^{l}$ for three possible $l \neq 1$, two of which are shown here (see Figure 4 in [20] for a diagram showing the full unstable manifold).

\begin{tabular}{c|c|c}
$k$ & $P_{j}^{k}$ & $\overline{W^{u}\left(P_{1}^{k}\right)} \supset$ \\
\hline 1 & $\left(0,0,0, \psi_{j}, \psi_{j}\right)$ & $P_{2}^{9}, P_{2}^{10}, P_{2}^{2}$ \\
2 & $\left(\psi_{j}, \psi_{j}, 0,0,0\right)$ & $P_{2}^{1}, P_{2}^{3}, P_{2}^{6}$ \\
3 & $\left(0,0, \psi_{j}, 0, \psi_{j}\right)$ & $P_{2}^{4}, P_{2}^{8}, P_{2}^{2}$ \\
4 & $\left(0, \psi_{j}, 0, \psi_{j}, 0\right)$ & $P_{2}^{3}, P_{2}^{5}, P_{2}^{10}$ \\
5 & $\left(\psi_{j}, 0,0,0, \psi_{j}\right)$ & $P_{2}^{6}, P_{2}^{4}, P_{2}^{9}$ \\
6 & $\left(0,0, \psi_{j}, \psi_{j}, 0\right)$ & $P_{2}^{7}, P_{2}^{5}, P_{2}^{2}$ \\
7 & $\left(0, \psi_{j}, 0,0, \psi_{j}\right)$ & $P_{2}^{6}, P_{2}^{8}, P_{2}^{10}$ \\
8 & $\left(\psi_{j}, 0,0, \psi_{j}, 0\right)$ & $P_{2}^{3}, P_{2}^{7}, P_{2}^{9}$ \\
9 & $\left(0, \psi_{j}, \psi_{j}, 0,0\right)$ & $P_{2}^{1}, P_{2}^{5}, P_{2}^{8}$ \\
10 & $\left(\psi_{j}, 0, \psi_{j}, 0,0\right)$ & $P_{2}^{1}, P_{2}^{7}, P_{2}^{4}$
\end{tabular}

Table 1

Representative points on the periodic orbits $P_{j}^{k}(j=1,2, k=1, \cdots, 10)$ in the unperturbed heteroclinic network (1). In all cases $\overline{W^{u}\left(P_{j}^{k}\right)} \supset P_{3-j}^{k}$ and $\overline{W^{u}\left(P_{1}^{k}\right)}$ contains the additional points listed. The three states $P_{2}^{k}$ in the unstable manifold can be reached via arbitrarily small perturbations to precisely one of the three synchronized oscillators of the state $P_{j}^{k}$.

$N-k$ at the other. The system (1) has an $\mathbf{S}^{1}$ symmetry (given by adding a constant to all phases) that allows one to characterize periodic orbits as group orbits. For the parameter values used in this letter $(N=5, \alpha=1.25, \omega=5$, $r=0.25)$ the only attractor for unperturbed (1) consists of twenty periodic orbits with symmetry $\mathbf{S}_{2} \times \mathbf{S}_{3}$ and unstable invariant manifolds connecting them. 
The periodic orbits involved are $\mathbf{S}^{1}$ orbits of $x_{j}=\left(0,0,0, \psi_{j}, \psi_{j}\right)$ with $j=1,2$ and permutations thereof (Table 1). We list a set of permutations $\sigma_{k}$ with $k=1, \cdots, 10$ that map the periodic orbit through $x_{j}$ onto the 10 different symmetric images and denote by $P_{j}^{k}$ the periodic orbit given by the $\mathbf{S}^{1}$ orbit of $\sigma_{k} x_{j}$ for $j=1,2$ and $k=1, \cdots, 10$. For the given parameters, $\psi_{1}=1.339$ and $\psi_{2}=0.799$ [20]. If we write $\Sigma=\bigcup_{i=1}^{10} \bigcup_{j=1}^{2} W^{u}\left(P_{j}^{k}\right)$ where $W^{u}(P)$ is the unstable manifold of the saddle periodic orbit $P$ then any randomly chosen initial condition approaches $\Sigma$; this manifests itself as a sequence of 'slowing down' switchings between different cluster states $P_{j}^{k}$. Fig. 1 shows a detail of the unstable manifold of one of the $P_{1}^{k}$.

For $N$ large (1) displays heteroclinic attractors between $\mathbf{S}_{k} \times \mathbf{S}_{N-k}$ symmetry states for a variety of $k$ with $N / 3<k \leq N / 2$ [18]; this gives rise to more complicated attracting heteroclinic networks that are currently under investigation by the authors. The unperturbed dynamics is such that after a transient period the system state is near one of the $P_{j}^{k}$ except for increasingly occasional short transitions. As $\Sigma$ is asymptotically stable [16] this is true even for small enough structural perturbations of the system. We exploit these features for our computational system. Typical initial conditions for the unperturbed system lead to an asymptotic state that is a slowing-down oscillation between $P_{1}^{k}$ and $P_{2}^{k}$ for some $k$ determined by the initial condition.

Trajectories of the perturbed system may visit many or all $P_{j}^{k}$ where $j$ always alternates between 1 and 2 and $k$ changes on a longer timescale [20]. This is because the unstable manifold of $P_{1}^{k}$ is two dimensional and only exceptional trajectories on this connect to $P_{2}^{l}$ with $k \neq l$. In the presence of noise we refer to the average time of switching between $P_{j}^{k}$ as the cycling time $T_{c}$; one can show that this scales as $-\ln \eta[16]$. The average time of switching between $P_{j}^{k}$ with different $k$ we refer to as the switching time $T_{s}$. Note that the order parameter, $\chi=\frac{1}{N}\left|\sum_{k=1}^{N} e^{i \theta_{k}}\right|$, often considered for such systems $[16,18]$ oscillates on the timescale $T_{c}$. Because this measure is invariant under permutations of the phases it is neither possible to detect switches nor compute $T_{s}$ using only $\chi$.

\section{Computation using cluster states}

One can view (1) as a computational system with five inputs $I_{i}(t)$ and twenty outputs $Y_{j}^{k}(\boldsymbol{\theta})=\min \left\{\mid \boldsymbol{\theta}-\boldsymbol{\phi} \|_{1}: \boldsymbol{\phi} \in P_{j}^{k}\right\}$ (where $\left.|\boldsymbol{\theta}|_{1}=\sum_{i}\left|\theta_{i}\right|\right)$, namely if $Y_{j}^{k}$ is small then the trajectory is close to $P_{j}^{k}$. Fig. 2 shows the statistics of $\delta$, the local minimum of the $Y_{j}^{k}$ over one cycle. On average $\langle\delta\rangle$ varies as the square root of the noise, reminiscent of the algebraic scaling of heteroclinic switching rates with noise level [21]. Defining the probability $P_{s}$ of switching per cycle we estimate $P_{s}=T_{c} / T_{s}$. Numerical simulations indicate that $T_{c} / T_{s}=O\left(\eta^{1 / 2}\right)$ as $\eta \rightarrow 0$; see Fig. 3. We define a state $\boldsymbol{\theta}$ to be close to the saddle $P_{j}^{k}$ if $Y_{j}^{k}(\boldsymbol{\theta})<\Delta$ 


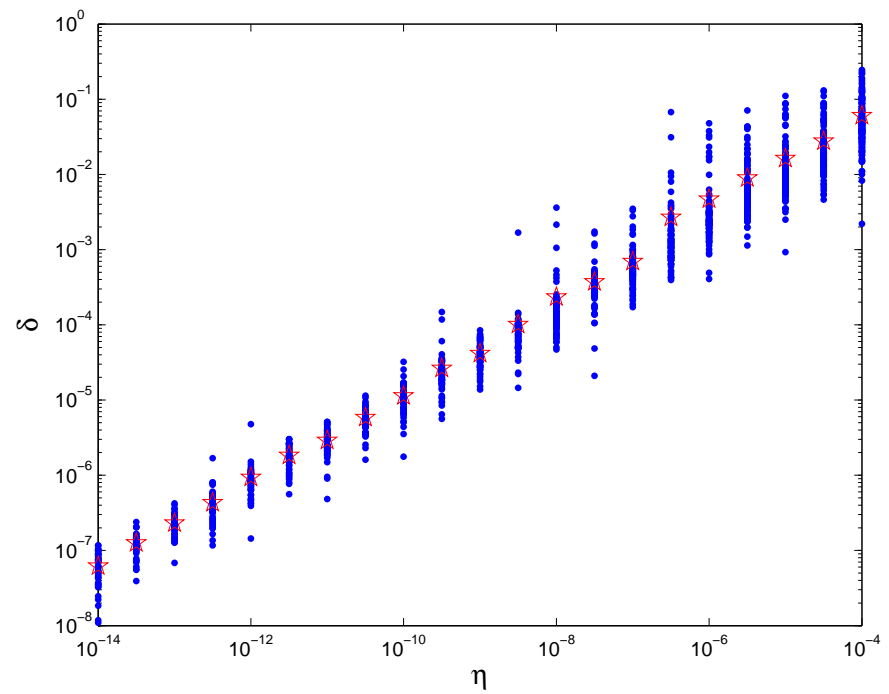

Fig. 2. Dots show the value of the smallest value $\delta$ of any $Y_{j}^{k}$ during a cycle time $T_{c}, \delta=\inf \left\{Y_{j}^{k}(t): j=1,2, k=1 . .10, t \in\left[t_{0}, t_{0}+T_{c}\right)\right\}$, in the presence of noise with amplitude $\eta$, after transients have decayed and for several random initial conditions. The mean closest approach (over 100 cycles) is marked ' $\times$ ' and scales as $\langle\delta\rangle=O(\sqrt{\eta})$.

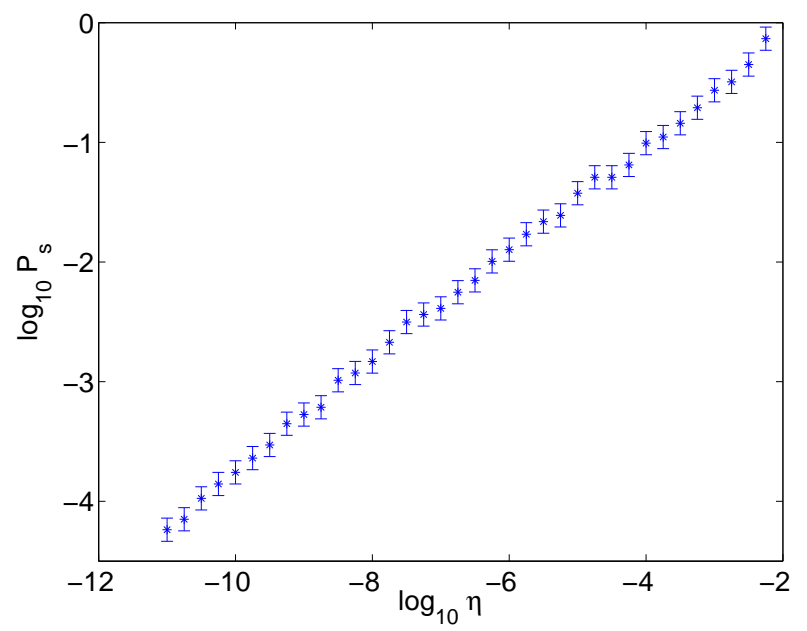

Fig. 3. Probability of switching $P_{s}$ at each cycle for specified $\eta$ with average over 100 trials. Error bars show two standard deviations; observe that $P_{s} \sim \sqrt{\eta}$.

where $\Delta=10 \sqrt{\eta}$. This ensures that all switches found in Fig. 2 are registered while it avoids false positive detections of switching, as (for instance) may occur on trajectories from $P_{1}^{1}$ to $P_{2}^{1}$ that pass very close to $P_{2}^{9}$ (see Fig. 1). One can then use simple logic gates to connect up outputs and inputs to form a computational system (see Fig. 4).

We now illustrate with an example the potential for computation in this system. We choose the saddles $P_{j}^{2}, P_{j}^{3}, P_{j}^{4}, P_{j}^{5}, P_{j}^{6}$ to represent states of a base 


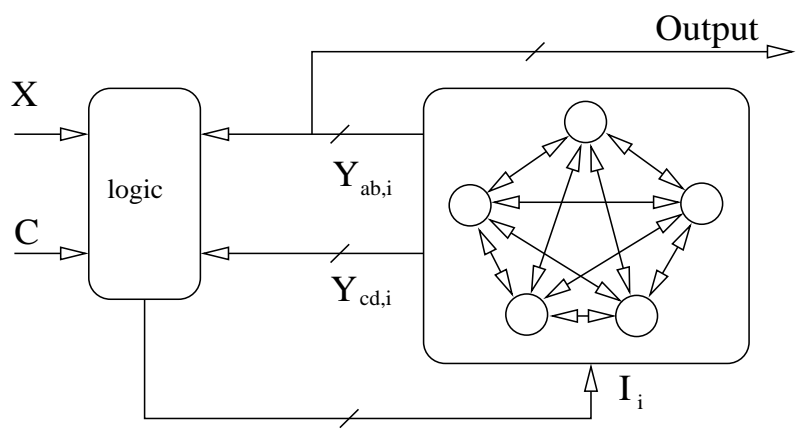

Fig. 4. Schematic wiring diagram for the counter using the five-oscillator system. The logic circuit steers the states around sequences of $P_{j}^{k}$ depending on $X, C$ and initial condition. The input to the system $X$ and clock $C$ are connected to one of the $I_{i}$ depending on which of the $Y_{j}^{k}$ are low.

5 counter and $P_{j}^{7}, P_{j}^{8}$ to represent states of a base 2 counter. Fig. 5 shows how computation is realised by clock pulses $C(t)$ and input pulses $X(t)$ that steer the system to the next state depending on current state. The inputs are steered to at most one of the oscillators [20]: we set $I_{i}(t)=C(t)$ if and only if $Y_{2}^{k}<\Delta$ and $i$ is one of the group of two synchronized oscillators, and $I_{i}(t)=X(t)$ if and only if $Y_{1}^{k}<\Delta$ and perturbation of oscillator $i$ directs the system to the next desired state of the counter; see Table 1. More precisely, we set all $I_{i}(t)$ to be zero except if $Y_{1}^{k}<\Delta$ then we set $I_{p(k)}(t)=X(t)$ where $p=[4,4,1,3,2,5,3,3,2,1]$ and if $Y_{2}^{k}<\Delta$ then we set $I_{\ell(k)}(t)=C(t)$ where $\ell=[4,1,3,2,1,3,2,1,2,1]$. Note that for $\Delta<0.1$, at most one of the $Y_{j}^{k}$ is less that $\Delta$.

Fig. 5 shows the network dynamics and the state as a function of the given clocking and input pulses. Fig. 6 illustrates that the system performs qualitatively the same for different parameter values. For different initial conditions, Fig. 6(d) shows that this system also works as a base two counter.

There is a trade-off between on the one hand the speed of computation and on the other hand its accuracy and sensitivity to noise. In particular the input amplitude $\epsilon$ gives a characteristic cycle time as does the noise amplitude $\eta$. Computation in the above system is reliable if $1 \gg \epsilon \gg \eta$ and the clock cycles lie between the cycle times associated with the two perturbation amplitudes.

\section{Discussion}

Many models have previously used oscillators or symmetries to perform computational tasks, for example [23-26]. The novelty in this work is that we demonstrate how one of the simplest possible types of coupling can give rise to robust attracting heteroclinic networks that one can exploit to perform 

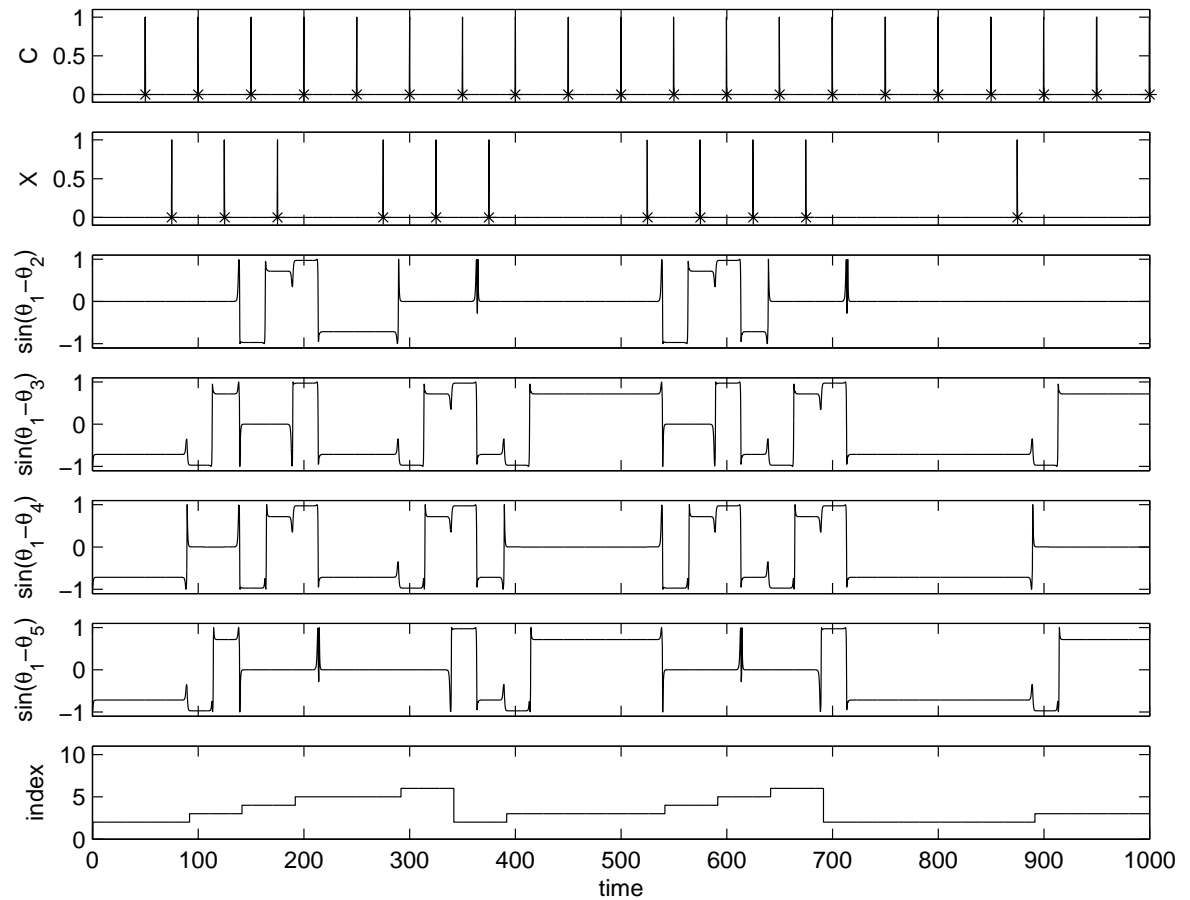

Fig. 5. Simulation of the counter for $C(t)$ and $X(t)$ as shown in the top graphs $\left(\eta=10^{-14}, \epsilon=10^{-10}\right)$ and an initial condition with $Y_{1}^{2}<\Delta$. The oscillator phase differences $\sin \left(\theta_{1}-\theta_{j}\right)$ are shown, while the state index (bottom graph) cycles one step through states $P_{1}^{k}$ where $k \in\{2,3,4,5,6\}$ each time an input on $X$ is received. The sequence of switches shown is realised by small perturbations to individual oscillators in the sequence $(4,1,3,2,5,4,1,3,2,5,4)$.

computations. In particular there is no need for careful tuning of coupling strengths. As in winnerless competition models $[1,8,9]$ the average phase differences in the globally coupled system change very little depending on the state of the system and the computation is performed by following natural trajectories within the system. By contrast, encoding by asymptotically stable states needs finite size perturbations to change state. We note the state of the system is not detectable from individual or 'mean' cell outputs but is encoded globally.

The unstable manifolds of dimension $>1$ allow several possible directions of switching from each state. This allows the network to scale to large $N$ without substantially increasing the minimum number of transitions needs to go from one state to any other. Moreover, the dynamics requires no careful tuning and works over a range of coupling parameters. We use two populations of processing elements (a) the globally coupled oscillators $\theta_{i}$ have the function of a 'memory/timing' circuit and (b) observables $Y_{j}^{k}$ from the system that detect presence of a particular cluster state. The latter are analogous to Kenyon Cells in the mushroom body that receive inputs from an insect $\mathrm{AL}[7,4,3,22]$ and decode a large dense set of states in a relatively low dimensional space to a sparse set of states in a higher dimensional space. 

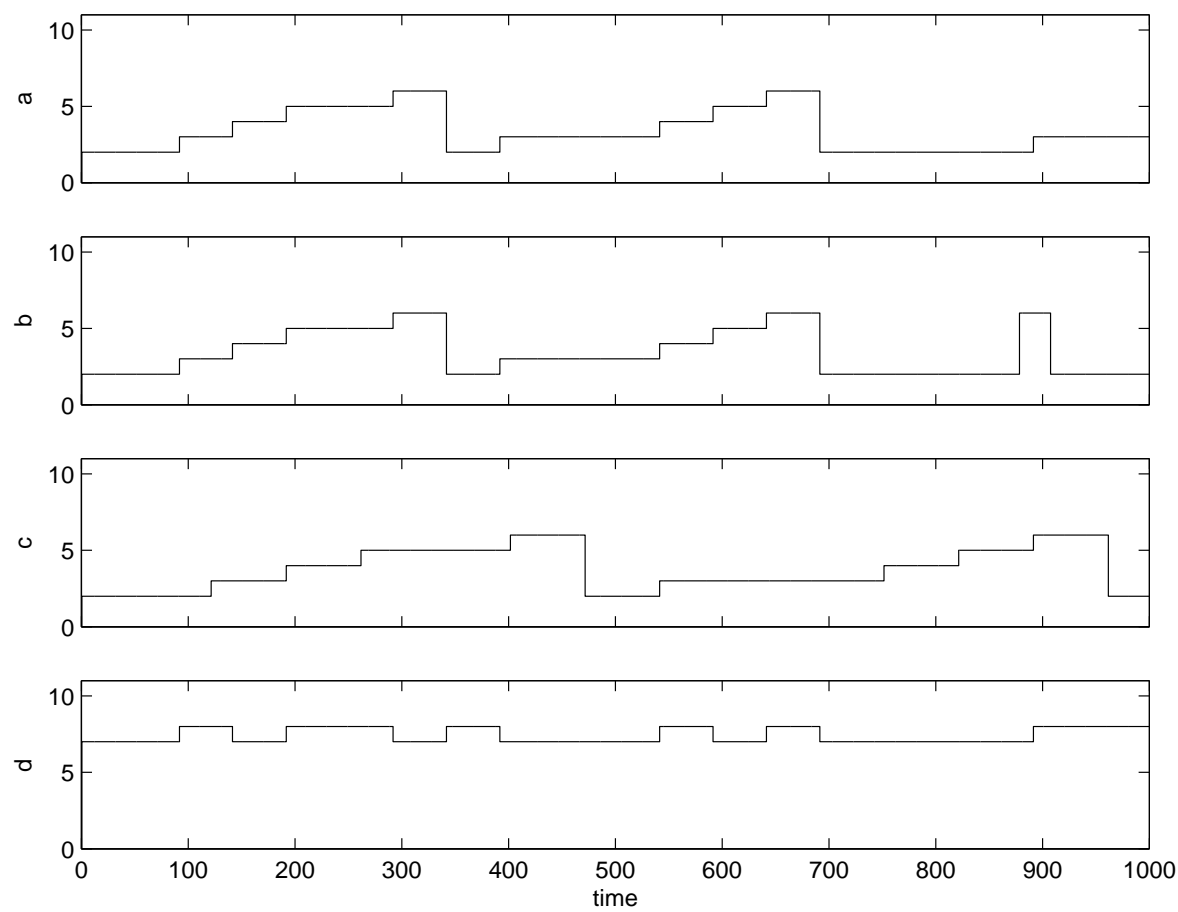

Fig. 6. Index giving the state of the counter for (a) the same inputs and initial condition as Fig. 5; (b) as for (a) but increased $\eta$ (observe the appearance of an erroneous switch just before $t=900$ ); (c) as for (a) also reliably functions with the clock rate slowed from 50 to 70 time units; (d) as for (a) but starting with an initial condition near state 7 ; this shows the presence of a second cycle in the network.

We believe that the simplicity of the global coupling used within the 'processing element' of our model is a positive feature. Although global coupling is only ever an approximation to real neural systems, the asymptotic stability of the heteroclinic attractor means that the dynamics will remain close even when the global coupling is perturbed. Moreover, there are evolutionary advantages for neural subsystem that attempt to approach symmetric coupling during development; their genetic coding can be much simpler than would be needed for a detailed 'wiring map'. The robustness of the dynamics of the unperturbed system means that the heteroclinic attractors used are model-independent and will permit similar computation using instead globally coupled systems of phase oscillators $[27,13]$, coupled ODE oscillators or even piecewise smooth systems of phase-resetting oscillators [28].

Similarly structured networks should also be possible between equilibria, at least in coupled higher dimensional systems, though phase oscillators are particularly amenable to work with. One feature we do require is at least 5 oscillators; namely this is necessary for unstable manifolds with dimension more than one [20]. The ideas presented should scale well on increasing $N$ beyond 5; the number of cluster states grows exponentially but the diameter of the heteroclinic network can stay small, meaning that rapid transition from one state to another is possible. However, the complexity of the heteroclinic networks 
rises very rapidly on increasing $N$ meaning that we do not presently understand higher $N$ systems to the degree that we can reliably perform similar computations on such systems.

Acknowledgements: We acknowledge very interesting discussions with Roman Borisyuk, David Corne, Gerhard Dangelmayr and especially Marc Timme concerning this research. The research of JB is supported by an EPSRC studentship and that of PA by a Leverhulme research fellowship. We are very grateful for the hospitality and financial support of the MPI für Strömungsforschung, Göttingen.

\section{References}

[1] M. Rabinovich, A. Volkovskii, P. Lecanda, R. Huerta, H.D.I. Abarbanel and G. Laurent, Dynamical encoding by networks of competing groups: winnerless competition. Phys. Rev. Lett. 87, 068102 (2001).

[2] G.B. Burg, C.G. Galizia, R. Brandt and H. Mustaparta, Digital atlasses of the antennal lobe in two species of tobacco budworm moths. J. Comp. Neurology 446, 123-134 (2002).

[3] D. Martinez and E. Hugues. A spiking neural network model of the locust antennal lobe: towards neuromorphic electronic noses inspired from insect olfaction. Proc. NATO ARW, Oct 1-2 2003, Coventry 2003 (2003).

[4] R. Huerta, T. Nowotny, M.G. Sanchez, H.D.I. Abarbanel and M.I. Rabinovich, Learning classification in the olfactory system of insects Neural Computation 16, 1601-1604 (2004).

[5] R.W. Friedrich and G. Laurent, Dynamics of olfactory bulb input and output activity during odor stimulation in zebrafish. J. Neurophysicol. 91, 2658-2669 (2004).

[6] R.W. Friedrich, C.J. Habermann and G. Laurent, Multiplexing using synchrony in the zebrafish olfactory bulb. Nature Neuroscience 7, 862-871 (2004).

[7] G. Laurent, M. Stopfer, R.W. Friedrich, M.I. Rabinovich, A. Volkovskii and H.D.I. Abarbanel, Odor encoding as an active dynamical process Ann. Rev. Neurosci. 24, 263-297 (2001).

[8] V. Aframovich, M. Rabinovich and P. Varona, Heteroclinic contours in neural ensembles and the winnerless competition principle. Int. J. Bifurcation and Chaos 14, 4 (2004).

[9] P. Seliger, L.S. Tsimring and M. Rabinovich, Dynamics-based sequential memory: Winnerless competition of patterns. Phys. Rev. E 67, 011905 (2003). 
[10] K. Otsuka, Y. Sato and J.-L. Chern, Clustering, grouping, self-induced switching and controlled dynamic pattern generation in an antiphase intracavity second-harmonic-generation laser. Phys. Rev. E. 56, 4765-4772 (1997).

[11] E.A. Viktorov, A.G. Vladimirov and P. Mandel, Symmetry breaking and dynamical independence in a multimode laser. Phys. Rev. E. 62, 6312-6317 (2000).

[12] M. Krupa, Robust heteroclinic cycles. J. of Nonlinear Sci. 7, 129-176 (1997).

[13] P. Ashwin and J.W. Swift, The dynamics of $\mathrm{n}$ weakly coupled identical oscillators. J. Nonlinear Sci. 2, 69-108 (1992).

[14] P. Ashwin and P. Chossat, Attractors for robust heteroclinic cycles with continua of connections. J. Nonlin. Sci. 8, 103-129 (1998);

[15] P. Ashwin and M. Field, Heteroclinic networks in coupled cell systems, Arch. Rational Mech. Anal. 148, 107-143 (1999).

[16] D. Hansel, G. Mato and C. Meunier, Clustering and slow switching in globally coupled phase oscillators. Phys. Rev. E 48, 3470-3477 (1993).

[17] D. Hansel, G. Mato and C. Meunier, Phase dynamics for weakly coupled Hodgkin-Huxley neurons. Europhys. Letts. 23, 367-372 (1993).

[18] H. Kori and Y. Kuramoto, Slow switching in globally coupled oscillators: robustness and occurrence through delayed coupling. Phys. Rev. E 63, 046214 (2001).

[19] H. Kori, Slow switching in a population of delayed pulse-coupled oscillators. Phys. Rev. E 68, 021919 (2003).

[20] P. Ashwin and J. Borresen, Encoding via conjugate symmetries for globally coupled oscillators. Phys. Rev. E 70, 026203 (2004).

[21] D. Armbruster, E. Stone and V. Kirk, Noisy heteroclinic networks. Chaos 13, 71-79 (2003).

[22] O. Perez-Orive, J. Mazor, G.C. Turner, S. Cassanaer, R.I. Wilson and G. Laurent, Oscillations and sparsening of odor representations in the mushroom body. Science 297, 359-365 (2002).

[23] R. Borisyuk, M. Denham, F. Hoppensteadt, Y. Kazanovich and O. Vinogradova, Oscillatory model of novelty detection. Network: Comp. Neural Syst. 12, 1-20 (2001).

[24] M. Breakspear, Perception of odors by a nonlinear model of the olfactory bulb. Int. J. of Neural Systems 11, 101-124 (2001).

[25] D.L. Rowe, Dynamic neural activity as chaotic itinerancy or heteroclinic cycles. Behavioural and Brain Sciences 24, 827-828 (2001). 
[26] M. Breakspear, J.R. Terry and K.J. Friston. Modulation of excitatory synaptic coupling facilitates synchronization and complex dynamics in a biophysical model of neuronal dynamics. Network: computation in Neural Systems 14, 703-732 (2003).

[27] F.C. Hoppensteadt and E. Izhikevich, Weakly Connected Neural Networks Springer (2000).

[28] M. Timme, F. Wolf and T. Geisel, Prevalence of unstable attractors in networks of pulse-coupled oscillators. Phys. Rev. Lett. 89, 154105 (2002). 